\title{
An Adaptive Questionnaire for Automatic Identification of Learning Styles
}

\author{
Esperance Mwamikazi, Philippe Fournier-Viger, Chadia Moghrabi, Adnen Barhoumi, \\ Robert Baudouin \\ Department of Computer Science, Université de Moncton, Canada \\ Department of Secondary Education and Human Resources, Université de Moncton, Canada \\ \{eem7706, philippe.fournier-viger, chadia.moghrabi\}@umoncton.ca, \\ bmhadnen@gmail.com, robert.baudouin@moncton.ca
}

\begin{abstract}
Learning styles refer to how a person acquires and processes information. Identifying the learning styles of students is important because it allows more personnalized teaching. The most popular method for learning style recognition is through the use of a questionnaire. Although such an approach can correctly identify the learning style of a student, it suffers from three important limitations: (1) filling a questionnaire is time-consuming since questionnaires usually contain numerous questions, (2) learners may lack time and motivation to fill long questionnaires and (3) a specialist needs to analyse the answers. In this paper, we address these limitations by presenting an adaptative electronic questionnaire that dynamically selects subsequent questions based on previous answers, thus reducing the number of questions. Experimental results with 1,931 questionnaires for the Myers Briggs Type Indicators show that our approach (Q-SELECT) considerably reduces the number of questions asked (by a median of $30 \%$ ) while predicting learning styles with a low error rate.
\end{abstract}

Keywords: adaptive questionnaire, association rules, neural networks, learning styles, Myers Briggs Type Indicator.

\section{Introduction}

Learning styles refer to how people acquire and process information [1, 2]. In education, knowing the learning styles of students is important because it allows to further personnalize interaction between teachers and students. It was shown in several studies that presenting information in a way that is adapted to the learning style of a learner facilitate learning [3].

Although several studies have been presented on how to perform learning style assessment, there are still several important limitations to current approaches. The first approach is used by e-learning systems that can provide adaptation according to the learning style of a learner. The approach consists of designing a software module that analyzes the learner's interactions with the system to detect the learning style [1, 4]. This approach 
has the benefit of being seamless for the learner. However, it suffers from a major drawback, namely that a random learning type is assigned initially to the learner and thus the system will initially guide and assist the learner according to that type. If the initial guess is incorrect, the system will thus interact with the learner according to the wrong learning style, which may have negative effect on learning. Furthermore, this interaction will continue until enough data is recorded to find the correct learning style [1].

The other main method for learning style assessment is to use a standardized questionnaire that a person has to fill out. A specialist then analyzes the answers to determine the correct learning style. The advantage of this approach is that the learning style of a person can be identified immediately. However, this approach suffers also from important limitations. First, questionnaires are usually very long. For example, the Myers-Briggs Type Indicator questionnaire discussed in this paper consists of more than 90 questions. This means that it is very time-consuming for a person to fill out the questionnaire. Second, long questionnaires have a negative effect on a person's motivation [4], which may lead to abandonning the test, skipping questions or answering falsely. This can furthermore provoke an incorrect learning style assessment, which may have undesirable consequences in future interactions [5]. For example, in the case of an e-learning system, if a learner does not answer the questionnaire correctly, the ensuing interactions with the system may be done according to a wrong learning style, which may have detrimental effect on learning. Third, using a questionnaire usually requires a specialist to analyze the learner answers and to determine the learning style.

In this paper, we address all the above limitations by presenting a novel learning style assessment approach, which takes the form of an adaptive electronic questionnaire. Our contributions are fourfold. First, the electronic questionnaire relies on an efficient algorithm PREDICT for predicting answers to upcoming questions based on associations between questions already answered and answers from previous users. Predicting answers allows skipping questions from the standardized questionnaire, thus reducing the number of questions to be answered by the learner. Second, the electronic questionnaire incorporates an efficient question selection algorithm QSELECT that analyzes associations between question answers to determine which questions should be asked first to minimize the number of questions asked when the aforementioned prediction algorithm is used. Third, once all questions have been answered or predicted, the electronic questionnaire uses a novel prediction algorithm to accurately predict a person learning style based on the answers and predicted answers. Fourth, we performed an extensive experimental study with 1,931 questionnaires for the assessment of the Myers-Briggs Type Indicator (MBTI). Results show that our approach reduces the number of questions presented to the user by a median of $30 \%$ while maintaining a low error rate in identifying the learning styles.

The rest of the paper is organized as follows. The Myers-Briggs Type Indicator model is presented in section 2. Section 3 discusses related work on adaptive questionnaires. In section 4 and 5, we respectively present the proposed electronic questionnaire and the experimental results. Finally, section 6 draws the final conclusions. 


\section{Myers-Briggs Type Indicator (MBTI)}

A popular personality inventory that has been used for more than 30 years is the Myers-Briggs Type Indicator (MBTI). This is a self-report questionnaire that identifies personality types using Carl Jung's personality type theory. A four-letter code is used to describe each individual's personality. It uses choice items to classify individuals into dichotomous preferences. One can either be extraverted (E) or introverted (I); sensing (S) or intuitive (I); thinking (T) or feeling (F) and finally be either judging (J) or perceiving $(\mathrm{P})$. Personality types are thus determined by the combination of these four dimensions. There are 16 four-letter codes that are possible (cf. Table 1). Descriptive outcomes of these codes or personality types help in one's classification [2, $6,7]$.

Table 1. The sixteen Myers-Briggs Type Indicators

\begin{tabular}{|l|l|l|l|}
\hline ISTJ & ISFJ & INFJ & INTJ \\
\hline ISTP & ISFP & INFP & INTP \\
\hline ESTP & ESFP & ENFP & ENTP \\
\hline ESTJ & ESFJ & ENFJ & ENTJ \\
\hline
\end{tabular}

Each type describes tendencies and reflects variations in individual attitudes and styles of decision-making. The E-I dimension (extraverted-introverted) focuses on whether an individual's world attitude is outwardly-oriented to other objects and individuals, or it is internally-oriented. The S-N dimension (sensing-intuitive), on the other hand, describes the perceptual style of an individual. Sensing refers to attendance to sensory stimuli while intuition entails analyzing stimuli and events. Based on the T-F dimension, thinking encompasses logical reasoning together with decision processes. This dimension further shows that feeling has to do with a personal, subjective, and value oriented approach $[6,7,8]$. The J-P dimension involves either a judging attitude and quick decision-making or perception that demonstrates more patience and information gathering prior to decision-making.

Some of the preferences are dominant and others are auxiliary and can be influenced by other dimensions. For example, the J-P dimension influences the two function preferences: $\mathrm{S}$ or $\mathrm{N}$ versus $\mathrm{T}$ or $\mathrm{F}[6]$.

The MBTI has its limitations. Its theoretical and statistical imports are limited by the application of dichotomous choice items [8]. The large number of questions to be answered can discourage users and cause them to fill out the questionnaire without much attention. Reducing the number of questions in a questionnaire has been a way to increase its efficiency $[4,9]$. 


\section{Related Work}

One major challenge in building a system that can adapt itself to a learner is giving it the capability of reducing the number of questions presented to the learner $[4,5,11]$. For instance, McSherry [9] reports that reducing the number of questions asked by an informal case-based reasoning system minimized frustration, made learning easier and increased efficiency.

Numerous researches on adaptive educational hypermedia systems have been conducted to minimize the number of questions asked to learners based on their capabilities and knowledge level [12 - 16] by using methods such as Item Response Theory $[12,14]$. Questions are initially categorized by their difficulty. The score that a learner obtains for each completed section determines the difficulty level of the next questions that would be asked and whether some questions should be skipped. Nevertheless, few researches have attempted to measure the impact of reducing the number of questions on the correct identification of the learner profile. The AH questionnaire in [4] relies on decision trees to reduce the number of questions and to classify students according to the Felder-Silverman model of learning styles. Its experimental results with 330 students show that it effectively predicts the learning styles with high accuracy and limited number of questions.

Petri et al. [17] proposed EDUFORM, a software module for the adaptation and dynamic optimization of questionnaire propositions for profiling learners online. This tool, based on probabilistic Bayesian modeling and on Abductive reasoning, reduced the number of questionnaire propositions (items) by 30 to 50 percent, while maintaining an error rate between 10 to 15 percent. Experimental results have shown that a significant reduction in the numbers of proposition in the questionnaires was often accompanied by a correct classification of individuals.

Even though these studies have shown that it was possible to reduce the length of questionnaire using adaptive mechanisms, and in the case of [4] to apply it to learning styles, none of these studies have been done with the MBTI model of learning styles. Furthermore, our work differs from [4] in two important ways. First, our approach allows computing likely answers to unanswered questions and those are also taken into account to predict the learning style of a learner. Second, our proposal is based on the novel idea of exploiting associations between answers and questions to predict answers and skip questions (by mining association rules and using neural networks).

\section{The Electronic Questionnaire}

In this section, we present our proposed electronic questionnaire for the automatic assessment of learning style. It comprises three components: (1) an answer prediction algorithm, (2) a dynamic question selection algorithm and (3) an algorithm to accurately predict a person's learning style based on both user supplied and predicted answers. 


\subsection{The answer prediction algorithm}

Let there be a questionnaire such as that of the MBTI. Let $Q=\left\{q_{1}, q_{2}, \ldots q_{n}\right\}$ be the set of multiple-choice questions from the questionnaire. Let $A\left(q_{i}\right)=\left\{a_{i, 1 \ldots} a_{i, m}\right\}$ denote the finite set of possible answers to a given question $q_{i}(1 \leq \mathrm{i} \leq n)$. Let $R=\bigcup_{i=1}^{n} A\left(q_{i}\right)$ be the set of all possible answers to all questions $q_{i}(1 \leq \mathrm{i} \leq n)$. A set of answers $U=\left\{u_{l}\right.$, $\left.u_{2} \ldots u_{k}\right\}$ is a set $U \subseteq R$ where there does not exist integers $a, b, x$ such that $u_{a}, u_{b} \in$ $A\left(q_{x}\right)$ and such that $a$ is different from $b$. A completed set of answers is a set of answers $U$ such that $|U|=n$. A partial set of answers is a set of answers $U$ such that $|\mathrm{U}|$ $<n$. An empty set of answers is a set of answers $U$ such that $|U|=0$. Given a set of answers $U$, a question $q_{x}$ is an unanswered question if $A\left(q_{x}\right) \cap U=\emptyset$. Otherwise, $q_{x}$ is an answered question. For a set of answers $U$ and a set of questions $Q$, Unanswered $(U, Q)$ denotes the set of unanswered questions, defined as Unanswered $(U, Q)$ $=\left\{q_{i} \mid q_{i} \in Q \wedge A\left(q_{i}\right) \cap U=\emptyset\right\}$. Intuitively, a "set of answers" would be the filled-out answers in a questionnaire that might be supplied by a user. It could be completed, partial, or empty.

Problem of answer prediction. Let $U$ be a partial set of answers. Let $q_{x}$ be an unanswered question such that $A\left(q_{x}\right) \cap U=\emptyset$. The problem of predicting the answer to $q_{x}$ is to determine the answer from $A\left(q_{x}\right)$ that the user would choose.

To address the above problem, we assume that we have a training set $T$ of completed sets of answers. This set is used to build a prediction model that is then used to predict answers for any unanswered question. In a set of answers $U$, we use the term predicted answer to refer to an answer that was predicted by the prediction model.

Building the prediction model. To build the prediction model, we rely on association rule mining, an efficient and popular method to discover associations between items in sets of symbols, originally proposed for market basket analysis [18]. In our context, the problem of association rule mining can be defined as follows. Given the training set $T$, the support of a set of answers $U$ is denoted as $\sup (U)$ and defined as the number of completed sets of answers in $T$ containing $U$, that is $\sup (U)=\mid\{V \mid V \in$ $T \wedge U \subseteq V\} \mid$. An association rule $X \rightarrow Y$ is a relationship between two sets of answers $X, Y$ such that $X \cap Y=\varnothing$. The support of a rule $X \rightarrow Y$ is defined as $\sup (X \rightarrow Y)=$ $\sup (X \cup Y) /|T|$. The confidence of a rule $X \rightarrow Y$ is defined as $\operatorname{conf}(X \rightarrow Y)=\sup (X \cup Y) /$ $\sup (X)$. The lift of a rule $X \rightarrow Y$ is defined as lift $(X \rightarrow Y)=\sup (X \rightarrow Y) /(\sup (X) \times$ $\sup (Y) /|T|^{2}$ ). The problem of mining association rules is to find all association rules in $T$ having a support no less than a user-defined threshold $0 \leq$ minsup $\leq 1$ and a confidence no less than a user-defined threshold $0 \leq \operatorname{minconf} \leq 1$ [18]. For instance, Figure 1 shows a set of completed answers $T$ (left) and some association rules found in $T$ for minsup $=0.5$, minconf $=0.5$ (right).

\begin{tabular}{|c|c|}
\hline ID & $\begin{array}{c}\text { sets of an- } \\
\text { swers }\end{array}$ \\
\hline$t_{1}$ & $\{a, b, c, e, f, g\}$ \\
\hline$t_{2}$ & $\{a, b, c, d, e, f\}$ \\
\hline$t_{3}$ & $\{a, b, e, f\}$ \\
\hline$t_{4}$ & $\{b, f, g\}$ \\
\hline
\end{tabular}$\rightarrow$\begin{tabular}{|c|c|c|c|}
\hline ID & Rules & Support & Confidence \\
\hline$r_{1}$ & $\{a\} \rightarrow\{e, f\}$ & 0.75 & 1 \\
\hline$r_{2}$ & $\{a\} \rightarrow\{c, e, f\}$ & 0.5 & 0.6 \\
\hline$r_{3}$ & $\{a, b\} \rightarrow\{e, f\}$ & 0.75 & 1 \\
\hline$r_{4}$ & $\{a\} \rightarrow\{c, f\}$ & 0.5 & 0.6 \\
\hline
\end{tabular}

Fig. 1. (a) A set of sets of answers and (b) some association rules found 
To build the prediction model, in our experiments with the MBTI questionnaire, we used minsup $=0.15$ and set minconf in the $[0.75,0.99]$ interval (the justification for these values are given in the experimental section). Choosing a high confidence threshold allows discovering only strong associations so that only those are used for prediction. Moreover, we also tuned the association rule mining algorithm to only discover rules of the form $X \rightarrow Y$ having a single item in the consequent, i.e. where $|Y|$ $=1$. The reason behind such a choice is that we are only interested in predicting one answer at a time rather than multiple answers together.

Performing a prediction. We now describe the algorithm for predicting the answer to an unanswered question $q_{\mathrm{z}}$ for a set of answers $U$, by using a set of association rules $A R$. Figure 2 shows the pseudocode of the prediction algorithm. It takes as input the question $q_{z}$, the current set of answers $U$ and the set of association rules $A R$. The algorithm first initializes a variable named prediction that will hold the final prediction and a variable highestMeasure to zero (line 1 to 2). Then, the algorithm considers each association rule $X \rightarrow Y$ from $A R$ such that the antecedent $X$ appears in $U$ and that the consequent $Y$ contains an answer to $q_{z}$ (line 3). For each rule, we calculate its usefulness for making a prediction, that we define as measure $=\operatorname{lift}(X \rightarrow Y) *|X|-$ $\mid$ Unanswered $(U, Q)|/| X \mid$ (line 4). A larger value of this measure is considered better. In this measure, a lift higher than 1 means a positive correlation between $X$ and $Y$, while a lift lower than 1 means a negative correlation. We multiply the lift by $|X|$ to give an advantage to rules matching with more answers from $U$ over rules matching with fewer answers. The term $|\operatorname{Unanswered}(U, Q)| /|X|$ is subtracted from the previous term so that previously predicted answers in $X$ have a negative influence on the measure (to reduce the risk of accumulating error by performing a prediction based on a previous prediction). The algorithm then selects the answer with the highest measure (line 9) as the prediction and adds it to the set of answers $U$ (line 9).

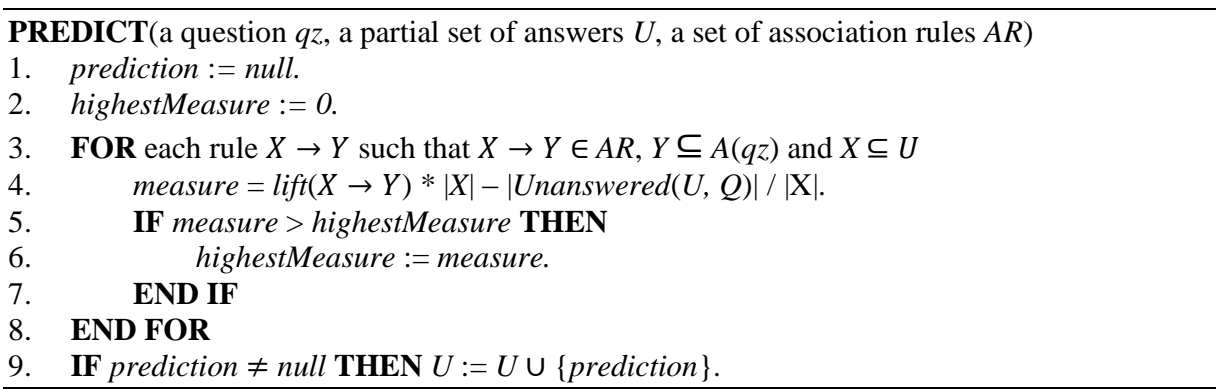

Fig. 2. The answer prediction algorithm

\subsection{The question selection algorithm}

We now describe Q-SELECT, the question selection algorithm of our electronic questionnaire that dynamically determines the order of questions. The pseudocode is given in Figure 3. The algorithm takes as input the set of association rules $A R$, previously extracted from the training set $T$. The algorithm first initializes the set of answers $U$ for the current user to $\varnothing$. Then, the algorithm scans in one pass association rules $A R$ to 
calculate dependencies of each question from $Q$. The set of dependencies of a question $q_{x}$ is denoted as dependencies $\left(q_{x}\right)$ and defined as the set of questions that can be used to predict an answer to $q_{x}$, i.e. dependencies $\left(q_{x}\right)=\left\{q_{z} \mid \exists X \rightarrow Y \in A R \wedge q_{\mathrm{z}} \in X\right.$ $\wedge Y \subseteq A(q)\}$. A question $q_{x}$ is said to be an independent question if no answer to that question can ever be predicted by the set of association rules $A R$, i.e. dependencies $\left(q_{x}\right)$ $=\varnothing$. If there are independent questions, the algorithm starts by asking them to the user (line 3 to 4). The reason behind such a priority is that answers to independent questions cannot be predicted. But, their answers may be used to predict answers for other questions. Then, for each unanswered question $q$, the algorithm calls PREDICT (cf. Section 4.1) in an attempt to predict an answer for $q$ (line 5).

After this loop, all independent questions and possible predictions have been exhausted. Next, the algorithm has to ask a question among the remaining unanswered questions. This is performed by a loop that continues until all questions have been answered (line 7). In this loop, the algorithm selects which question should be asked next. To make this choice, the algorithm estimates the number of questions that can be unlocked for each unanswered question if it was answered. The set of questions that a question $q$ can unlock is denoted as unlockable $(q)$ and defined as unlockable $(q)=\left\{q_{z} \mid \exists X \rightarrow Y \in A R \wedge \exists z \in A\left(q_{z}\right) \wedge z \in Y \wedge X \subseteq U \cup A(q) \wedge A(Q) \cap X \neq \emptyset\right\}$. The algorithm calculates this set for each unanswered question. This can be done by scanning the set of association rules once (line 8). Then the algorithm asks the question that can unlock the maximum number of questions according to the previous definition (line 9). Thereafter, for each unanswered question, the algorithm calls PREDICT to use the answer provided by the user to attempt to make a prediction (line 10). The WHILE loop then continue in the same way until no unanswered question remains. When the loop terminates, for each question $q$ in $Q$, the set of answers $U$ contains an answer from $A(q)$, which has either been answered by the user or predicted.

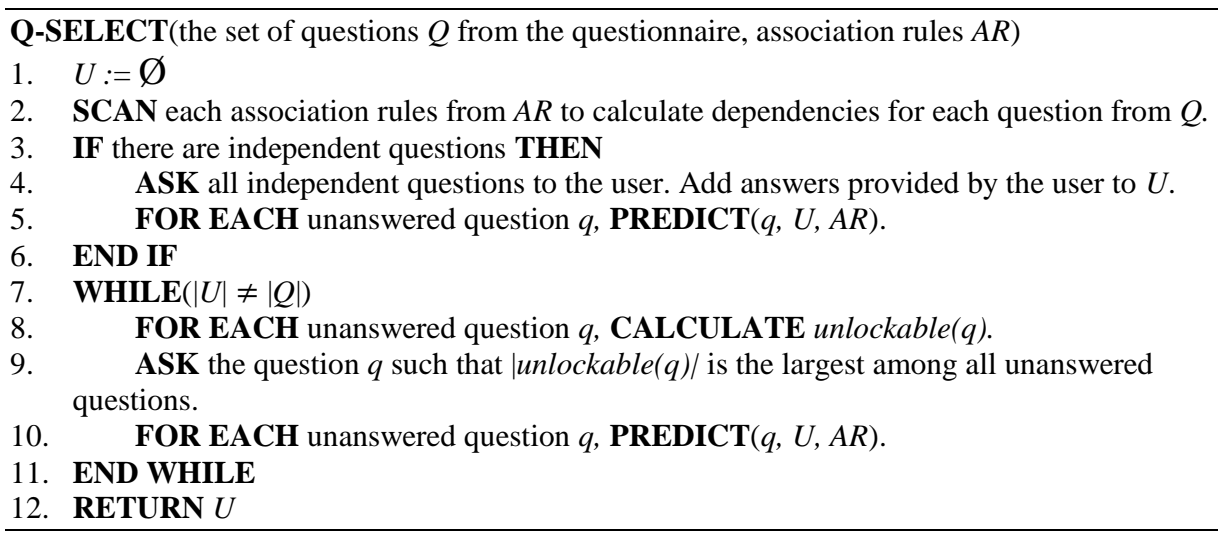

Fig. 3. The question selection algorithm 


\subsection{The learning style prediction algorithm}

We now describe how the electronic questionnaire automatically identifies the learning style of a user based on supplied and predicted set of answers.

The MBTI questionnaire evaluates each dimension (EI, JP, TF and SN) by a distinct subset of questions. Thus, we split the questionnaire into four sets of questions representing each dimension. A prediction algorithm is applied to each subset (dimension) to identify the individual's preference based on available answers. Finally, preferences in all four dimensions are combined to establish the learning style of the user.

Identifying the preference of a person in each dimension is essentially a classification problem. It is achieved, in our system, by a single layer feed-forward neural network, among the most common neural network architectures (it connects the input and output neurons directly, rather than connecting them through an intermediate layer). Neural networks are generally more accurate than other classifiers [9]. We trained a neural network for each dimension using 1,000 filled questionnaires (cf. experimental section). Thereafter, for each new user, the set of answers produced by the question selection algorithm (cf. Section 4.2) is used as input by the networks. The number of input neurons for each network is the number of questions for the corresponding dimension. The MBTI questionnaire uses 21 questions to assess the EI dimension, 23 questions for TF, 25 questions for $\mathrm{SN}$, and 23 questions for JP. There is a single binary neuron as the output of each network because of the dichotomic nature of each dimension. Neural networks are built in MATLAB with the following parameters: activation function $=$ TANSIG, performance function $=$ MSE, number of iterations $=1000$, the algorithm used for the training phase was TRAINLM with Goal $=0$, Minimum gradient $=1 \mathrm{e}^{-10}$ and Max-fail $=6$.

\section{Experimental Results}

A database of 1,931 MBTI completed questionnaires was provided by Prof. Robert Baudouin, an experienced specialist of the MBTI technique at the Université de Moncton. We used 1,000 samples for training and 931 for testing and evaluating the electronic questionnaire. The questionnaire is implemented using Java and MATLAB. The goal of the experiment was to measure how the elimination of questions influences the error rate. Since our questionnaire is dynamic and can eliminate a different number of questions for each questionnaire, the number of questions eliminated was measured using the median. Preliminary experimentation showed that minsup values lower than 0.15 did not increase accuracy. Thus, to vary the number questions eliminated (predicted), we instead varied the minconf threshold (in the [0.75, 0.99] interval). The error rate for a dimension is the number of questionnaire where the predicted preference is correct, divided by the total number of questionnaires in the test set.

Experimental results are shown in Table 2. The baseline error rates (when no questions are eliminated) are 3.7\%, $4.9 \%, 6 \%$ and $5 \%$ for the EI, SN, TF and JP dimensions, respectively. We limited our studies to error rates to no more than $12 \%$. The 
maximum number of questions that can be eliminated within this bound is shown in the last row of each column of Table 2. For EI, SN, TF and JP, the median number of questions eliminated is respectively $6,9,8$, and 5, with an error rate of $9.9 \%, 11.8 \%$, $12 \%$ and $11.7 \%$. The combined median number of questions eliminated is 28 , which represents $30.4 \%$ of the MBTI questionnaire. It is important to note that the above numbers are medians. In many cases, individuals had more questions eliminated than the median. For example, Table 3 shows the distribution of questions eliminated for the TF dimension. Although the median is eight questions, nine questions were eliminated for 292 individuals, and less than eight questions were eliminated for only 231 individuals.

Given the error rates from Table 2, the probability of predicting incorrectly four preferences for a particular user is only $0.02 \%$. The probability of predicting three erroneous preferences is $0.6 \%$. The probability of predicting two erroneous preferences is $6.66 \%$, while the probability of predicting one erroneous preference is $33 \%$, and the probability of a perfect prediction is $60 \%$. We note that the combined probability of having no errors or only one error is more than $92 \%$.

Table 2. Number of questions eliminated and the corresponding error rate

\begin{tabular}{|c|c|c|c|c|}
\hline $\begin{array}{c}\text { Median number of } \\
\text { questions eliminated } \\
\text { (predicted) }\end{array}$ & $\begin{array}{c}\text { Error rate for } \\
\text { EI }\end{array}$ & $\begin{array}{c}\text { Error rate for } \\
\text { SN }\end{array}$ & $\begin{array}{c}\text { Error rate } \\
\text { for TF }\end{array}$ & $\begin{array}{c}\text { Error rate for } \\
\text { JP }\end{array}$ \\
\hline \hline 0 & $3.7 \%$ & $4.9 \%$ & $6 \%$ & $5 \%$ \\
\hline 1 & $4.6 \%$ & $5,3 \%$ & $7,8 \%$ & $6 \%$ \\
\hline 2 & $5.2 \%$ & $6,3 \%$ & $8,4 \%$ & $7.1 \%$ \\
\hline 3 & $7.5 \%$ & $6,6 \%$ & $9,1 \%$ & $8.6 \%$ \\
\hline 4 & $7.7 \%$ & $7,5 \%$ & $10 \%$ & $10 \%$ \\
\hline 5 & $7.9 \%$ & $9 \%$ & $10,7 \%$ & $11.7 \%$ \\
\hline 6 & $9.9 \%$ & $10,5 \%$ & $11,7 \%$ & \\
\hline 7 & & $11,1 \%$ & $11,7 \%$ & \\
\hline 8 & & $11,7 \%$ & $12 \%$ & \\
\hline 9 & & $11,8 \%$ & & \\
\hline
\end{tabular}

We compared the Q-SELECT algorithm results with those obtained by a C4.5 decision tree $[19,20]$. Table 4 shows the comparable error rates of both methods for the highest number of questions eliminated by the Q-SELECT algorithm. It can be noticed that the error rates generated by the decision tree are two to four percent higher for each of the four preferences. 
Table 3. Number of questions eliminated per questionnaire for the TF dimension

\begin{tabular}{|c|c|c|}
\hline $\begin{array}{c}\text { Number of questions } \\
\text { eliminated }(x)\end{array}$ & $\begin{array}{c}\text { Number of questionnaires } \\
\text { (individuals) }\end{array}$ & $\begin{array}{c}\text { Number of questionnaires in the }[x, 10] \\
\text { interval }\end{array}$ \\
\hline \hline 1 & 0 & $931(100 \%)$ \\
\hline 2 & 11 & $931(100 \%)$ \\
\hline 3 & 7 & $920(99 \%)$ \\
\hline 4 & 3 & $913(98 \%)$ \\
\hline 5 & 11 & $910(98 \%)$ \\
\hline 6 & 14 & $899(97 \%)$ \\
\hline 7 & 185 & $885(95 \%)$ \\
\hline 8 (median) & 348 & $700(75 \%)$ \\
\hline 9 & 292,00 & $352(38 \%)$ \\
\hline 10 & 60 & $60(6 \%)$ \\
\hline & & \\
\hline
\end{tabular}

Table 4. Comparative results for Q-SELECT and Decision Tree

\begin{tabular}{|c|c|c|c|c|}
\hline & $\begin{array}{c}\text { Error rate for EI } \\
\text { (6 questions) }\end{array}$ & $\begin{array}{c}\text { Error rate for SN } \\
\text { (9 questions) }\end{array}$ & $\begin{array}{c}\text { Error rate for TF } \\
\text { (8 questions) }\end{array}$ & $\begin{array}{c}\text { Error rate for JP } \\
\text { (5 questions) }\end{array}$ \\
\hline \hline Q-SELECT & $9.9 \%$ & $11.8 \%$ & $12 \%$ & $11.7 \%$ \\
\hline $\begin{array}{c}\text { Decision } \\
\text { Tree }\end{array}$ & $12.35 \%$ & $13.21 \%$ & $16.54 \%$ & $13.74 \%$ \\
\hline
\end{tabular}

\section{Conclusion}

Standardized questionnaires for learning style identification are long, time-consuming and require human intervention to determine an individual's learning style. To address this issue, we presented an adaptive electronic questionnaire. It incorporates an efficient answer prediction algorithm PREDICT for predicting answers to unanswered questions based on associations between answers. We also presented Q-SELECT, an algorithm that reorders questions and minimizes the number of those presented, based on associations between questions. Experimental results with 1,931 questionnaires filled for the Myers Briggs Type Indicators show that our approach considerably reduces the number of presented question.

The combined median number of questions eliminated is 28 , which represents 30.4 $\%$ of the MBTI questionnaire. The combined probability of having no errors or only one error out of four preferences is more than $92 \%$. We also note that the Q-SELECT algorithm gave better results than the decision tree for all the preference types. 


\section{References}

1. Graf, S.: Adaptivity in Learning Management Systems Focusing on Learning Styles. Ph.D. Thesis, Vienna University of Technology, Vienna (2007)

2. Felder, R. M.: Matters of Style. ASEE Prism, 6(4), 18-23 (1996)

3. Felder, R. M., Brent, R.: Understanding student differences. J. of Engineering Education. 94(1), 57-72 (2005)

4. Ortigosa, A., Paredes, P., Rodríguez, P.: AH-questionnaire: An adaptive hierarchical questionnaire for learning styles. Computers \& Education. 54(4), 999-1005 (2010)

5. García, P., Amandi, A., Schiaffino, S., Campo, M.: Evaluating Bayesian Networks' Precision for Detecting Students' Learning Styles. Computers \& Edu., 49 (3), 794-808. (2007)

6. El Bachari, E., Abelwahed, E. H., El Adnani, M.: Design of an Adaptive E-Learning Model Based on Learner's Personality. Ubiquitous Computing Comm. J. 5(3), 27-36 (2010)

7. Boyle, G. J.: Myers-Briggs Type Indicator (MBTI): Some psychometric limitations. Australian Psychologist. 30(1), 71-74 (2009)

8. Francis, L. J., Jones, S. H.: The Relationship between Myers-Briggs Type Indicator and the Eysenck Personality Questionnaire among Adult Churchgoers. Pastoral Psychology. 48(5), 377-386 (2008)

9. McSherry, D.: Increasing dialogue efficiency in case-based reasoning without loss of solution quality. In: Proc. $18^{\text {th }}$ Intern. Joint. Conf. Artif. Intell., pp. 121-126 (2003)

10. Zhang, G. P.: Neural Networks for Classification: A Survey. IEEE Transactions on Systems, Man, and Cybernetics. 30(4), 451-462 (2000)

11. Abernethy, J., Evgeniou, T., Vert, J.-P.: An optimization framework for adaptive questionnaire design. Technical Report, INSEAD, Fontainebleau, France (2004)

12. Baylari, A., Montazer, G.: Design a personalized e-learning system based on item response theory and artificial neural network approach. Expert Syst. Appl. 36(4), 8013-8021 (2009)

13. Papanikolaou, K. A., Grigoriadou, M., Magoulas, G. D., Kornilakis, H.: Towards New Forms of Knowledge Communication: the Adaptive Dimension of a Web-based Learning Environment. Computers \& Education. 39(4), 333-360 (2002)

14. Chen, C. M., Lee, H. M., Chen, Y. H.: Personalized e-learning system using item response theory. Computers \& Education, 44(3), 237-255 (2005)

15. Brusilovsky, P., Millán, E.: User models for adaptive hypermedia and adaptive educational systems. In: Proc. The adaptive web 2007, pp. 3-53, Springer, Heidelberg (2007)

16. Xanthou, M.: An Intelligent Personalized e-Assessment Tool Developed and Implemented for a Greek Lyric Poetry Undergraduate Course. Electron. Journal of e-Learning. 11(2), 101-114 (2013)

17. Nokelainen, P., Niemivirta, M., Tirri, H., Miettinen, M., Kurhila, J., Silander, T. Bayesian Modeling Approach to Implement an Adaptive Questionnaire. In: Proc. ED-MEDIA 2001, pp. 1412-1413, AACE, Chesapeake (2001)

18. Agrawal, R., Imieliński, T., Swami, A.: Mining association rules between sets of items in large databases. ACM SIGMOD Record, 22( 2), 207-216, ACM (1993)

19. Quinlan, J. R.: C4. 5: programs for machine learning. Vol. 1. Morgan Kaufmann (1993)

20. Russell, S.J., Norvig, P.: Artificial Intelligence: A Modern Approach, Third Edition. Pearson (2010) 